\title{
Cross-correlation between the soft X-ray background and SZ Sky
}

\author{
L.-M. Cheng ${ }^{1,2}$, X.-P. Wu ${ }^{1}$, and A. Cooray ${ }^{3}$ \\ 1 National Astronomical Observatories, Chinese Academy of Sciences, Beijing 100012, PR China \\ 2 Department of Astronomy, Beijing Normal University, Beijing 100875, PR China \\ ${ }^{3}$ California Institute of Technology, Mail Code 130-33, Pasadena, CA 91125, USA
}

Received 24 March 2003 / Accepted 30 September 2003

\begin{abstract}
While both X-ray emission and Sunyaev-Zel'dovich (SZ) temperature fluctuations are generated by the warm-hot gas in dark matter halos, the two observables have different dependence on the underlying physical properties, including the gas distribution. A cross-correlation between the soft X-ray background (SXRB) and the SZ sky may allow an additional probe on the distribution of warm-hot gas at intermediate angular scales and redshifts complementing studies involving clustering within SXRB and SZ separately. Using a halo approach, we investigate this cross-correlation analytically. The two contributions are correlated mildly with a correlation coefficient of $\sim 0.3$, and this relatively low correlation presents a significant challenge for its detection. The correlation, at small angular scales, is affected by the presence of radiative cooling or preheating and provides a probe on the thermal history of the hot gas in dark halos. While the correlation remains undetectable with CMB data from the WMAP satellite and X-ray background data from existing catalogs, upcoming observations with CMB missions such as Planck, for the SZ side, and an improved X-ray map of the large scale structure, such as the one planned with DUET mission, may provide a first opportunity for a reliable detection of this cross-correlation.
\end{abstract}

Key words. cosmology: theory - cosmology: cosmic microwave background - galaxies: intergalactic medium cosmology: large-scale structure of universe $-\mathrm{X}$-rays: diffuse background

\section{Introduction}

The best current census of baryons in the universe conducted at high and low redshifts reveals that a considerably large fraction of baryons in the local universe is still missing (Fukugita et al. 1998). Hydrodynamical simulations of structure formation suggest that the missing baryons may exist in the form of warm-hot intergalactic medium (WHIM) with temperatures of $T \sim 10^{5}-10^{7} \mathrm{~K}$ (Cen \& Ostriker 1999; Davé et al. 2001). This arises because baryons can be gravitationally heated and adiabatically compressed when they fall into large-scale structures, including collapsed dark matter halos. However, while there has been observational evidence for presence of missing baryons associated with large-scale structures at low redshifts, which includes the degree-scale X-ray filaments (Scharf et al. 2000; Zappacosta et al. 2002), the soft X-ray excess emission in the vicinity of nearby clusters (Nevalainen et al. 2003; Kaastra et al. 2003), the resonant absorption lines of local warm gas towards distant AGNs/QSOs (e.g. Fang et al. 2002; Nicastro et al. 2002, 2003), the statistical confidences related to these detections remain poor and majority of the baryons still escape the direct detection.

In addition to $\mathrm{X}$-ray emission, missing baryons manifest themselves through the inverse-Compton scattering of cosmic

Send offprint requests to: L.-M. Cheng,

e-mail: clm@class1.bao.ac.cn microwave background (CMB) photons. The latter is known as the Sunyaev-Zel'dovich (SZ) effect. Indeed, massive groups and clusters that serve as a reservoir of the WHIM are very luminous sources in both X-ray and SZ maps. The diffuse WHIM in poor groups and filamentary structures associated with the large-scale "cosmic web", may not be strong enough to allow direct detection in individual cases. This gas, however, may make a significant contribution to the SXRB and CMB temperature fluctuation background related to the SZ effect, provided that the known sources such as AGNs and nearby, rich clusters can be removed from the SXRB and SZ sky. Recall that a considerably fraction (80-90\%) of the SXRB has been resolved into discrete sources (see Xue \& Wu 2003 for a recent summary). To extract the presence of missing baryons and reconstruct the content and the distribution, one needs to rely on certain statistical approaches. These include the two-point auto-correlation function of the SXRB or SZ sky, or the crosscorrelation of SXRB and/or SZ map with galaxies, groups and clusters (e.g. Soltan et al. 2001, 2002; Zhang \& Pen 2003; Wu $\&$ Xue 2003). Another possibility discussed here is the crosscorrelation between maps of SXRB and SZ effect.

As is known, X-ray measurement is a sensitive probe of the hot gas that is located in and near central regions of dark matter halos, mainly groups and clusters, and in the local universe. Recall that the X-ray emissivity within the framework of bremmstrahlung is proportional to the square of the 
electron density, and the X-ray flux is inversely proportional to the square of the distance from us. This compares to the SZ effect, which reveals a more extended distribution of hot gas in dark halos as a result of the linear dependence of the electron density and out to high redshifts because of the rather weak dependence on distance. Therefore, it is expected that the crosscorrelation between the SXRB from diffuse gas and the SZ sky may allow one to explore the distribution and evolution of the gas at intermediate scales and redshifts.

To estimate the extent to which this cross-correlation is present and whether it can be detected, we make use of a halo approach (e.g., Cooray \& Sheth 2002) with gas assumed to either trace dark matter or follow the $\beta$-profile. We then investigate how non-gravitational heating and radiative cooling can modify the cross power spectrum following discussions related to these effects in Wu \& Xue (2003). We also discuss another interesting application of the SXRB and SZ cross-correlation. Since the SXRB traces the square of the number density of electrons, while the SZ contribution is only sensitive to the integrated number density, any clumping of the gas distribution, such that the mean of the squared gas distribution is higher than the square of the mean, the cross-correlation between SXRB and SZ will be augmented at angular scales corresponding to the clumping of gas. Thus, any reliable detection of the SXRB and SZ cross-correlation can then be used to understand the clumped nature of the gas distribution, which is an important aspect of the WHIM and cannot be easily obtained by other means.

To illustrate our results, we adopt a flat cosmological model $(\Lambda \mathrm{CDM})$ with the best fit parameters determined by WMAP (Spergel et al. 2003): $\Omega_{\mathrm{M}}=0.27, \Omega_{\Lambda}=0.73, \Omega_{\mathrm{b}} h^{2}=$ $0.0224, h=0.71, \sigma_{8}=0.84$ and $n_{\mathrm{s}}=0.93$. During the preparation of this work, a paper by Diego et al. (2003) appeared claiming no detection of the cross-correlation between CMB data, as obtained by the WMAP satellite, and X-ray background data obtained by a map of the ROSAT soft X-ray emission. This lack of CMB-SXRB cross-correlation may be attributed to either a smaller value of $\sigma_{8}$ or the relatively weak signals at large angular scales. While the initial claim for a detection has disappeared, we suggest that the upcoming Planck mission will allow a first detection of the SZ-SXRB cross-correlation. The advantage over current data is that with Planck, one can use multifrequency information to extract a separate map of the large scale structure SZ effect (Cooray et al. 2000), which can then be cross-correlated directly with an X-ray map. The current approach, involving WMAP data, is not likely to be useful given that the fluctuations related to SZ is dominated by the primordial fluctuations of CMB related to physics at last scattering, such as the acoustic peak structure.

\section{SXRB and SZ cross-correlation}

\subsection{SXRB and $S Z$ effect}

The thermal SZ effect along the direction $\boldsymbol{\theta}$ due to the hot gas inside a halo can be evaluated following

$$
\frac{\Delta T(\boldsymbol{\theta})}{T_{\mathrm{CMB}}}=g_{v}(x) y(\boldsymbol{\theta})
$$

$$
\begin{aligned}
& y(\boldsymbol{\theta})=\int n_{\mathrm{e}} \sigma_{T}\left(\frac{k T}{m_{\mathrm{e}} c^{2}}\right) \mathrm{d} \chi \\
& g_{\nu}(x)=\frac{x^{2} e^{x}}{\left(e^{x}-1\right)^{2}}\left(4-x \operatorname{coth} \frac{x}{2}\right),
\end{aligned}
$$

where $x=h_{p} v / k T_{\mathrm{CMB}}$ is the dimensionless frequency, $T_{\mathrm{CMB}}$ is the temperature of the present $\mathrm{CMB}$, and the integral is performed along the line of sight, $\chi$.

In terms of bremmstrahlung emission, the X-ray surface brightness distribution in direction $\boldsymbol{\theta}$ is given by

$S_{\mathrm{X}}(\boldsymbol{\theta})=\frac{1}{4 \pi(1+z)^{4}} \int n_{\mathrm{e}} n_{\mathrm{H}} \Lambda(T, Z) \mathrm{d} \chi$,

where $n_{\mathrm{H}}$ is the number density of hydrogen, and $\Lambda(T, Z)$ is the cooling function in a given energy band that is calculated using the Raymond-Smith (1977) code with a metallicity of $Z=0.3 Z_{\odot}\left(t / t_{0}\right)$, and $t_{0}$ is the present age of the universe. The mean SXRB brightness $S_{\mathrm{X}}$ is calculated through

$\left\langle S_{\mathrm{X}}\right\rangle=\int \mathrm{d} z \frac{\mathrm{d} V}{\mathrm{~d} \Omega \mathrm{d} z} \int \mathrm{d} M \frac{\mathrm{d}^{2} N}{\mathrm{~d} M \mathrm{~d} V}\left[\frac{L_{X}(M, z)}{4 \pi D_{L}^{2}(z)}\right]$,

where $L_{X}(M, z)$ is the total $\mathrm{X}$-ray luminosity of a halo of mass $M$ at redshift $z, D_{\mathrm{L}}$ is the luminosity distance, and $\mathrm{d}^{2} N / \mathrm{d} M \mathrm{~d} V$ is the comoving number density of dark halos. For the latter we adopt the mass function of Jenkins et al. (2001).

We follow two approaches to describe the electron distribution within halos. First, gas is assumed to trace dark matter such that

$n_{\mathrm{e}}=\frac{f_{\mathrm{b}}}{\mu_{\mathrm{e}} m_{\mathrm{p}}} \rho_{\mathrm{DM}}$,

in which we have introduced the universal baryon fraction $f_{\mathrm{b}}=\Omega_{\mathrm{b}} / \Omega_{\mathrm{M}}$, and $\mu_{\mathrm{e}}=1.13$ is the mean electron weight. We adopt the universal density profile as suggested by cosmological numerical simulations (Navarro et al. 1995; NFW) to describe the dark matter distribution, $\rho_{\mathrm{DM}}$, in halos

$\rho_{\mathrm{DM}}(r)=\frac{\delta_{\mathrm{ch}} \rho_{\mathrm{crit}}}{\left(r / r_{\mathrm{s}}\right)\left(1+r / r_{\mathrm{s}}\right)^{2}}$,

where $\delta_{\mathrm{ch}}$ and $r_{\mathrm{s}}$ are the characteristic density and length of the halo, respectively, which can be fixed through the so-called concentration parameter $c=r_{\text {vir }} / r_{\mathrm{s}}$ using the empirical fitting formula found by numerical simulations (Bullock et al. 2001)

$c=\frac{10}{1+z}\left(\frac{M}{2.1 \times 10^{13} M_{\odot}}\right)^{-0.14}$.

The second approach involves the $\beta$-model with

$n_{\mathrm{e}}=\frac{n_{\mathrm{e} 0}}{\left[1+\left(r^{2} / r_{c}^{2}\right)\right]^{3 / 2}}$.

We fix the $\beta$ value to $\beta=2 / 3$ and specify the core radius by $r_{\mathrm{c}}=0.1 r_{\mathrm{vir}}$. The normalization $n_{\mathrm{e} 0}$ is determined using the universal baryon fraction. Finally, we need to specify the temperature profile $T(r)$. For the first model, $T(r)$ can be obtained in principle by solving the equation of hydrostatic equilibrium, while for the second one either the gravitational potential of 
dark matter or the equation of state for the gas must be given in order to work out $T(r)$. For simplicity, we now assume an isothermal model for the gas distribution in both approaches and specify its temperature in terms of virial theorem (e.g. Bryan \& Norman 1998):

$k T=1.39 \mathrm{keV} f_{T}\left(\frac{M}{10^{15} M_{\odot}}\right)^{2 / 3}\left(h^{2} E^{2} \Delta_{\mathrm{c}}\right)^{1 / 3}$

where $\Delta_{\mathrm{c}}$ denotes the overdensity parameter, and $f_{T}$ is the normalization factor which will be fixed to be $f_{T}=0.8$ in the following evaluation. While the isothermality may certainly introduce some uncertainties in our numerical predictions, this assumption should be good enough to provide an approximate estimate of to what extent the SXRB and SZ sky may correlate with each other at small scales. As an example, the typical central electron densities in the $\beta$-model turn to be $6.6 \times 10^{-3} \mathrm{~cm}^{-3}$ and $0.015 \mathrm{~cm}^{-3}$ for a group of $M=5 \times 10^{13} M_{\odot}$ and a rich cluster of $M=5 \times 10^{15} M_{\odot}$, respectively, at present epoch.

\subsection{Power spectra}

Following halo approach to large scale structure clustering, the angular cross power spectrum of the SXRB-SZ correlation can be separated into the Poisson term $C_{\ell}^{P}$ and the clustering term $C_{\ell}^{C}$ :

$$
\begin{aligned}
C_{\ell}^{P}= & g_{v}(x) \int_{0}^{z_{\mathrm{dec}}} \mathrm{d} z \frac{\mathrm{d} V}{\mathrm{~d} z \mathrm{~d} \Omega} \int_{M_{\min }}^{\infty} \mathrm{d} M \frac{\mathrm{d}^{2} N(M, z)}{\mathrm{d} M \mathrm{~d} V} \\
& \times\left|y_{\ell}(M, z) s_{\ell}(M, z)\right|,
\end{aligned}
$$

and

$$
\begin{aligned}
C_{l}^{C}= & g_{v}(x) \int_{0}^{z_{\mathrm{dec}}} \mathrm{d} z \frac{\mathrm{d} V}{\mathrm{~d} z \mathrm{~d} \Omega} P(k=\ell / D, z) \\
& \times\left[\int_{M_{\min }}^{\infty} \mathrm{d} M \frac{\mathrm{d}^{2} N(M, z)}{\mathrm{d} M \mathrm{~d} V} b(M, z) y_{\ell}(M, z)\right] \\
& \times\left[\int_{M_{\text {min }}}^{\infty} \mathrm{d} M \frac{\mathrm{d}^{2} N(M, z)}{\mathrm{d} M \mathrm{~d} V} b(M, z) s_{\ell}(M, z)\right],
\end{aligned}
$$

where $z_{\mathrm{dec}} \approx 1000$ is the CMB photon decoupling redshift, $D$ is the comoving angular diameter distance to the halo of mass $M$ at $z$, and $y_{\ell}$ and $s_{\ell}$ are the Fourier transforms of the Compton $y$-parameter and the SXRB fluctuation, $s(\theta) \equiv$ $\left[S_{\mathrm{X}}(\theta)-\left\langle S_{\mathrm{X}}\right\rangle\right] /\left\langle S_{\mathrm{X}}\right\rangle$, respectively. Here, $b(M, z)$ is the bias parameter, for which we use the analytic prescription of Mo \& White (1996). We take the minimum halo mass to be $M_{\min }=$ $10^{12} M_{\odot}$. Our final result is unaffected by this choice since both the SZ sky and SXRB are dominated by massive halos, and the shallower gravitational potential of galactic halos can hardly preserve the hot gas because of preheating or feedback process of star formation. Replacing $g_{v}(x) y_{\ell}$ (or $s_{\ell}$ ) by $s_{\ell}$ [or $g_{v}(x) y_{\ell}$ ] in Eqs. (11) and (12), we can get power spectra of the SXRB (or SZ map). Finally, the cross-correlation coefficient is calculated by

$r_{\ell}=\frac{C_{\ell}^{\mathrm{SXRB}-\mathrm{SZ}}}{\sqrt{C_{\ell}^{\mathrm{SZ}} C_{\ell}^{\mathrm{SXRB}}}}$,

which quantitatively indicates the strength of the crosscorrelation.

\subsection{Non-gravitational effect}

Presence of non-gravitational effect on the distribution and global properties of hot gas in groups and clusters has been firmly established over the past few years. Here we use a simple phenomenological approach to demonstrate how the SXRB-SZ cross power spectrum is modified by the non-gravitational processes such as preheating or radiative cooling, both of which tend to flatten the gas distribution in the central regions of groups and clusters and become indistinguishable in the explanation of the observed X-ray properties of groups and clusters. We refer the reader to the recent work of Xue \& Wu (2003) for a set of analytic models of preheating and cooling processes and the predicted properties of the WHIM. The basic procedures are summarized below:

(1) Preheating model. We begin with the gas distribution predicted by self-similar model and then raise the entropy distribution of the WHIM in dark halos, defined as $S=k T / n_{\mathrm{e}}^{2 / 3}$, by a certain level $S_{\text {floor }}$ which is fixed to $120 \mathrm{keV} \mathrm{cm}{ }^{2}$ as suggested by X-ray measurements of groups and clusters (Ponman et al. 1999; but see Ponman et al. 2003). The new distribution of the WHIM can be obtained by solving the equation of hydrostatic equilibrium.

(2) Cooling model. A certain amount of WHIM in the central regions of groups and clusters will be removed from the hot phase if its cooling time is shorter than the cosmic age, and higher-entropy gas at large radii would then flow inward. Following the prescription of Voit \& Bryan (2001) and Wu \& Xue (2002), we first estimate the total cooled mass of WHIM within the cooling radius by combining energy conservation and setting the cooling time to equal the cosmic age. We then derive the new distribution of the WHIM after cooling by solving the equation of hydrostatic equilibrium under the conservation of total baryonic mass and entropy.

\subsection{Gas clumping}

In order to make an estimate of the effect related to WHIM clumping spatially, we define the clumping parameter as

$C(r)=\frac{<n_{\mathrm{e}}^{2}(r)>}{<n_{\mathrm{e}}(r)>^{2}}$,

which describes the excess gas distribution related to the mean. Note in this definition we ignored the unhomogeneity of temperature. Since the X-ray emission scales as square of the number density, any clumping affects the SXRB while SZ remains unaffected unless the density variation is completely compensated by the temperature modulation. While one can potentially use the SXRB information alone to understand clumping, this requires prior knowledge on the mean of the gas distribution and this is readily available from the SZ side. Thus, a combined study of SZ and SXRB, such as the cross-correlation of the two, can be used to extract information related to any potential clumping of the gas distribution.

\section{Results}

In Fig. 1, we show the power spectra of SXRB and SZ effect; the two are calculated in the energy band $0.5-2.0 \mathrm{keV}$ and at the 


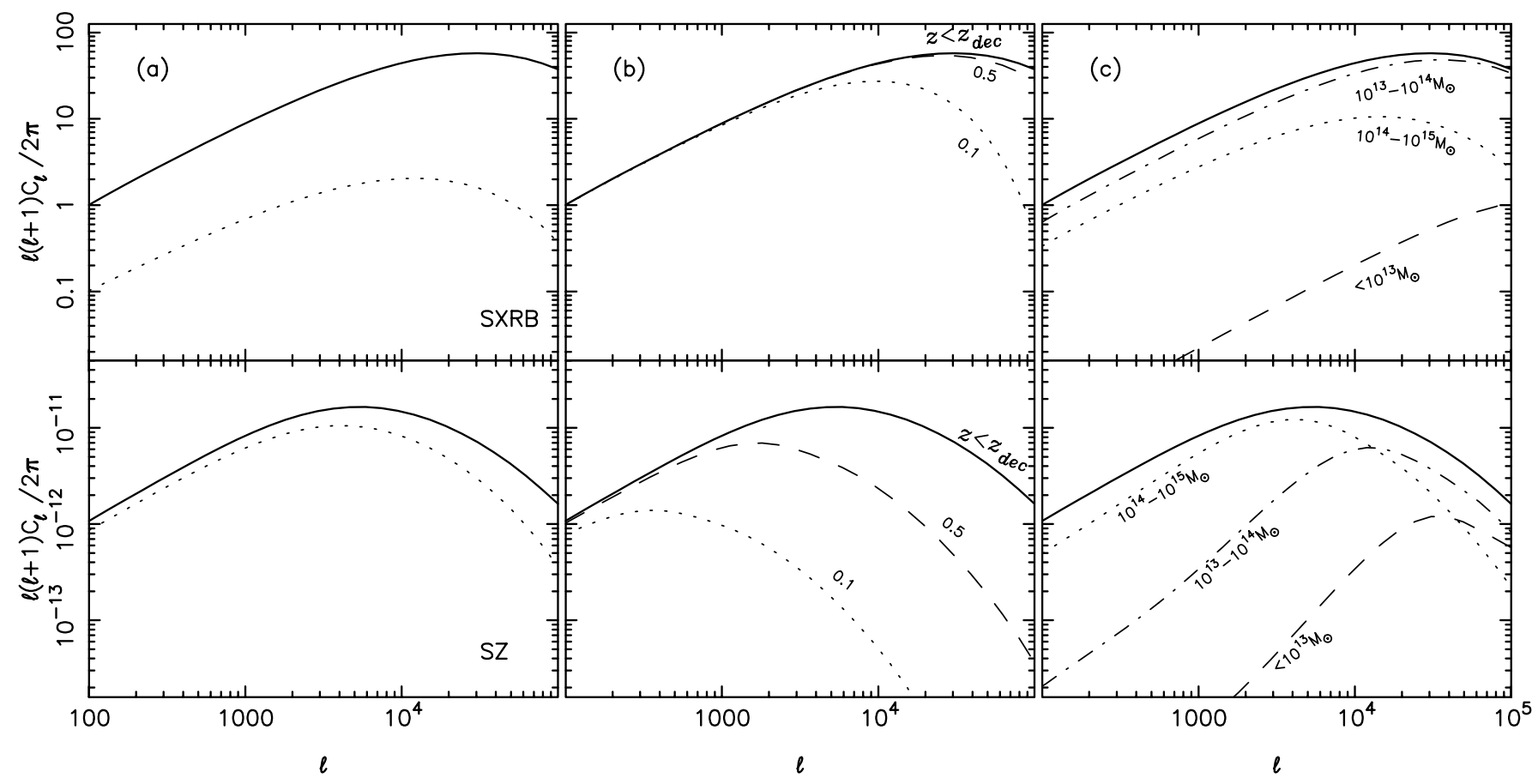

Fig. 1. The angular power spectra of the SXRB (upper panel) and the SZ sky (lower panel) measured in the energy band $0.5-2.0 \mathrm{keV}$ and at frequency $v=30 \mathrm{GHz}$, respectively. In a), two models are assumed for the gas distribution inside dark halos: the self-similar model (NFW-like model; solid lines) and $\beta$ model with $\beta=2 / 3$ and $r_{\mathrm{c}}=0.1 r_{\mathrm{vir}}$ (dotted lines). In $\mathbf{b}$ ), we show contributions from halos at different redshifts while in $\mathbf{c}$ ), we show contributions from halos separated in mass.

frequency $v=30 \mathrm{GHz}$, respectively. Our result also holds for other observing frequencies when the frequency dependence term $g(v)$ in the SZ effect is correspondingly corrected. It is apparent that the SXRB shows a stronger power at $\ell>10^{4}$ than the SZ map, which arises simply from the different dependence of the SXRB and SZ effect on the gas density: The former varies as $n_{\mathrm{e}}^{2}$ while the latter goes as $n_{\mathrm{e}}$. Employment of a $\beta$ model with $\beta=2 / 3$ leads to a significant drop of the power spectra at large $\ell$, especially for the SXRB, which can be attributed to the relatively flat core radius in the gas distribution when compared to the NFW-like profile.

In Fig. 1b, we illustrate contributions of halos in different redshift ranges to the SXRB and SZ power spectra. As it is expected, the SXRB power spectrum at $\ell<10^{5}$ is dominated by nearby halos within $z<0.5$, and high-redshift halos only make a minor contribution to the power spectrum at $\ell$ up to $10^{5}$, which is due to the well-known inverse dependence of $\mathrm{X}$-ray flux on the square of cosmic distances to halos. On the contrary, because the SZ effect reflects the thermal energy of hot gas intrinsic to halos, the SZ power spectrum, at small angular scales corresponding to $\ell>10^{3}$, is governed by halos at $z>0.5$.

In Fig. 1c, we show the dependence of SXRB and SZ power spectra on halo mass. It appears that massive clusters with $M>10^{14} M_{\odot}$ determine the SZ power spectrum at $\ell<10^{4}$, and at sub arcminute scales when $\ell \sim 10^{5}$, poor clusters and groups with $M \sim 10^{13}-10^{14} M_{\odot}$ starts to contribute. This compares to the SXRB power spectrum, when $\ell>10^{2}$, that is entirely dominated by low mass groups. Inclusion of nongravitational heating or radiative cooling processes may alter this mass-dependence (see Fig. 3).
In Fig. 2, we show the angular cross power spectrum between the SXRB and the SZ effect calculated with NFW and $\beta$ models. Note that we have dropped the negative sign in the power spectrum arising from $g_{v}(x)<0$ for $v=30 \mathrm{GHz}$. The cross power spectrum in the case of NFW model peaks at $\ell \approx 10^{4}$, between peak locations of SXRB and SZ power spectra. The same conclusion also applies to the $\beta$ model, though the peak now appears at a slightly smaller value of $\ell$. Moreover, at $\ell$ values beyond $10^{2}$, the contribution related to the halo-halo clustering term is insignificant. The contribution of halos at different redshifts and with different masses to the SXRB-SZ cross power spectrum is shown in Figs. $2 b$ and c. Essentially, at $\ell<10^{4}$, the power spectrum is governed by nearby clusters and groups with masses in the range $M>10^{14} M_{\odot}$ within $z=0.5$. In the range of $10^{4}<\ell<10^{5}$, groups with masses in the range of $10^{13}<M<10^{14} M_{\odot}$ and at redshifts greater than 0.5 begin to dominate the power spectrum. Unlike the SXRB power spectrum, the cross-correlation of SXRB and SZ contributions at $\ell>10^{4}$ is sensitive to clusters and groups at high redshifts. This, of course, is a combined result of the different redshift dependence of the SXRB and SZ power spectra.

The effects of preheating and radiative cooling on the power spectra are illustrated in Fig. 3, together with the autocorrelation power spectra of SXRB and SZ sky. As compared with the prediction of self-similar models, inclusion of preheating or cooling leads to a significant modification of the shape of the cross power spectrum at small angular scales, which can be attributed to the flattened distribution of the hot gas in the central regions of dark halos produced by preheating and/or 


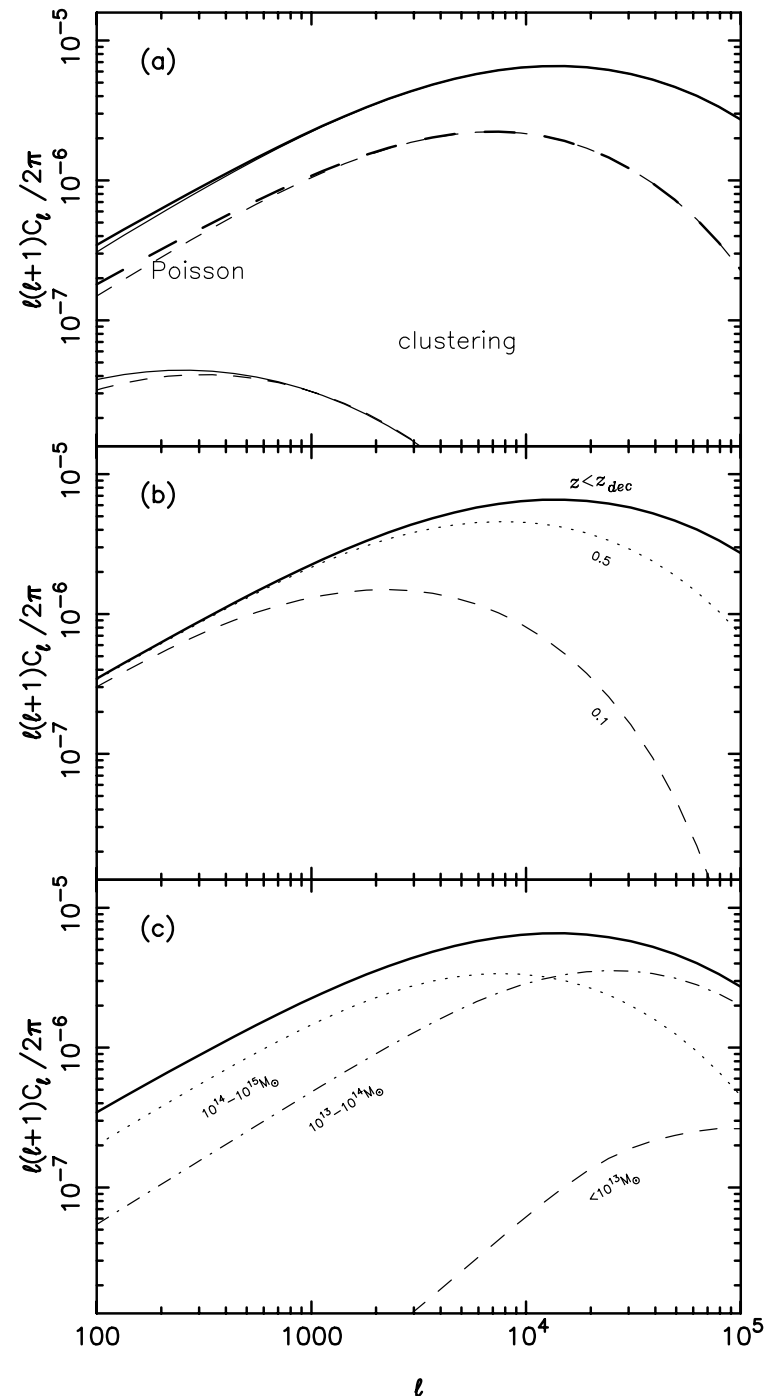

Fig. 2. a) The expected power spectra of the SXRB-SZ crosscorrelation for the NFW-like profile (solid lines) and $\beta$ model (dashed lines). Contributions from the Poisson and clustering distributions of dark halos are explicitly shown (thin lines). In b) and c), contributions from halos at different redshifts and varying masses are shown, respectively.

radiative cooling. Actually, the effect of nongravitational process is somewhat equivalent to the excision of a certain inner region in groups and clusters in the evaluation of SZ effect, which always leads to a decrease of the SZ power-spectrum amplitude at small scales (Komatsu \& Seljak 2002). While our predicted power spectra of the SZ sky and SXRB with preheating or cooling are roughly consistent with the previous studies of da Silva et al. (2001), Zhang \& Wu (2003), Zhang \& Pen (2003), and Wu \& Xue (2003), the extent to which the SZ (or SXRB) power spectrum is modified by nongravitational effect is still uncertain. For example, using high resolution hydrodynamic simulations White et al. (2002) found that nongravitational effect on the SZ power spectrum is only minor. A combination of the future high-resolution data of SXRB-SXRB, SZ-SZ and/or SXRB-SZ correlations at different scales may allow us to clarify the issue.

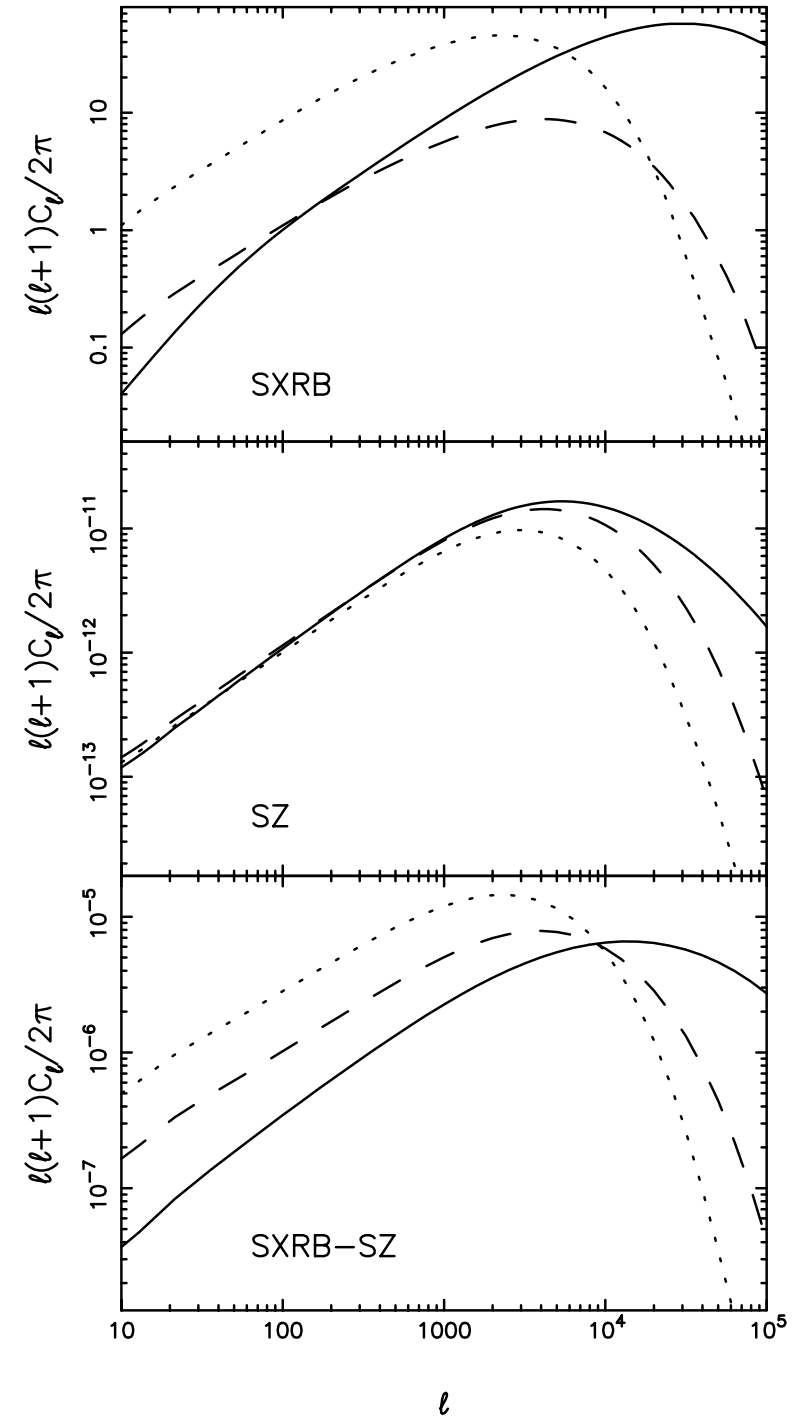

Fig. 3. Effect of non-gravitational processes on the power spectra of SXRB-SXRB (top panel), SZ-SZ (middle panel) and SXRB-SZ (lower panel) correlations. The results of self-similar model, preheating model and cooling model are displayed by solid, dotted and dashed lines, respectively.

In Fig. 4, we demonstrate the expected cross power spectra before and after the clumping correction. The major uncertainty in such an exercise is the clumping profile $C(r)$, which is poorly constrained by both current numerical simulations and $\mathrm{X}$-ray observations. Guided by the result of $N$-body simulations (e.g. Ghigna et al. 2000; Zentner \& Bullock 2003) that the spatial distribution of the clumps can be approximated by power laws, we take a toy model for $C(r)$ which has a powerlaw form of $C(r)=\left(\frac{r}{r_{v i \mathrm{ix}}}\right)^{\alpha}$. We fix the power law index $\alpha$ through $C(r)=1.5$ at $r=0.2 r_{\text {vir }}$. Of course, the oversimplification of this model can only give us a sense of how the cross power spectra might be affected by the gas clumping. We find that the difference is minor for our toy model. To obtain a substantial modification, which will be detectable, the clumping factor should be at the level of $\sim 10$.

In Fig. 5, we plot the correlation coefficient between SZ and SXRB. As shown, the correlation coefficient has a value 


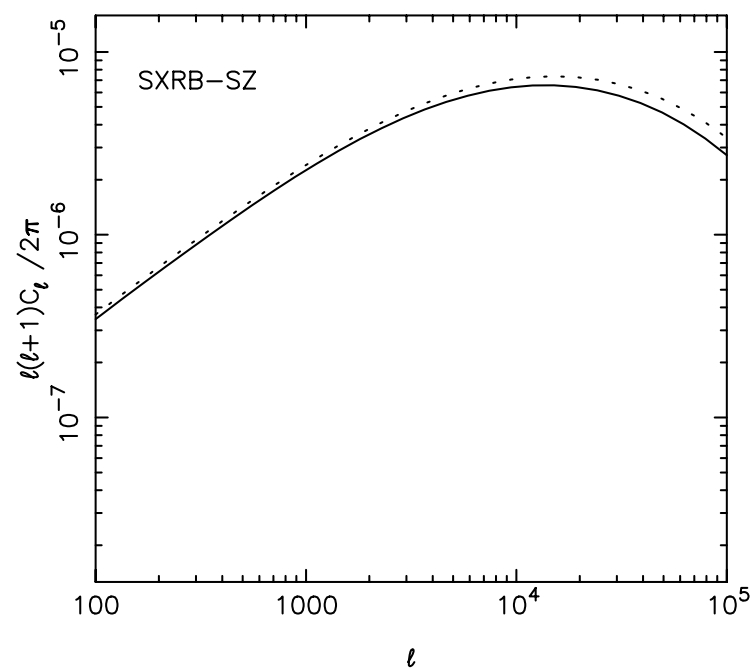

Fig. 4. Effect of the WHIM clumping on the cross power spectra. Solid line corresponds to the WHIM distribution described by the NFW profile without clumping structure $[C(r)=1]$, and dotted line represents that with a clumping characterized by $C(r)=\left(r / r_{\text {vir }}\right)^{-0.25}$.

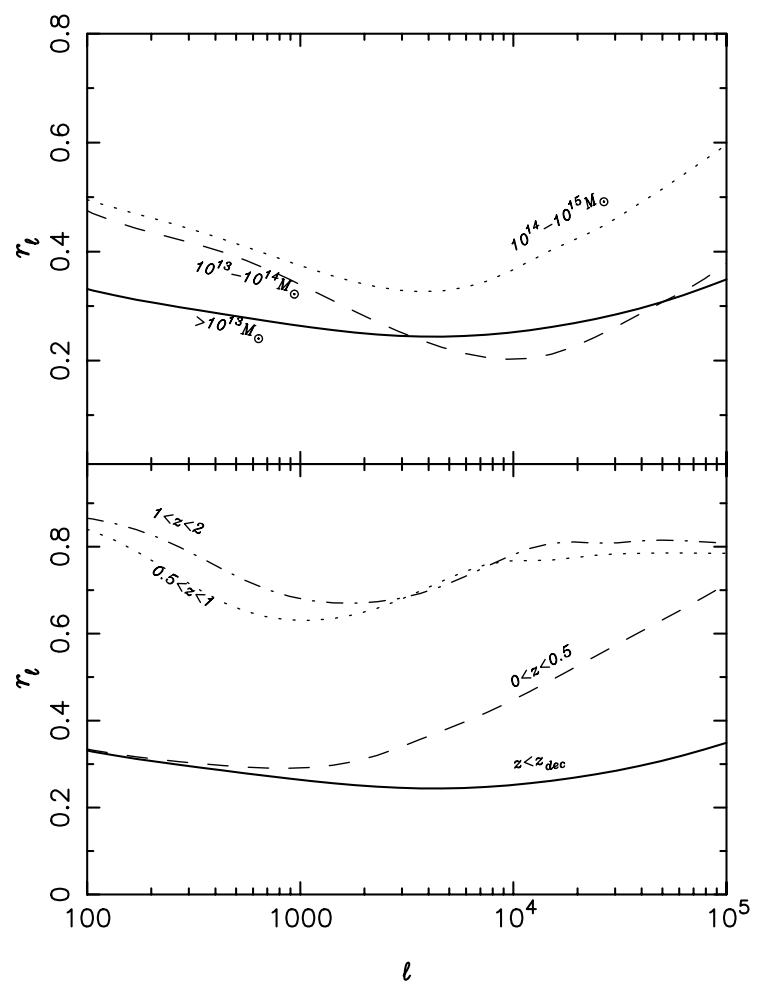

Fig. 5. The cross-correlation coefficient for the SZ effect and SXRB. With no separation of contributions in either redshift or mass-space, the correlation is at the level of $\sim 0.3$.

of $\sim 0.3$, suggesting that SZ and SXRB are not well correlated. While this is partly due to mismatches in redshifts where contributions arise, the correlation coefficient can be as high as 0.9 when one considers contributions only at high redshifts. The correlation coefficient is mostly constant when $\ell$ ranges over three decades of magnitude between 100 an $10^{5}$, suggesting that the scale dependence between SZ and SXRB is not drastically different.

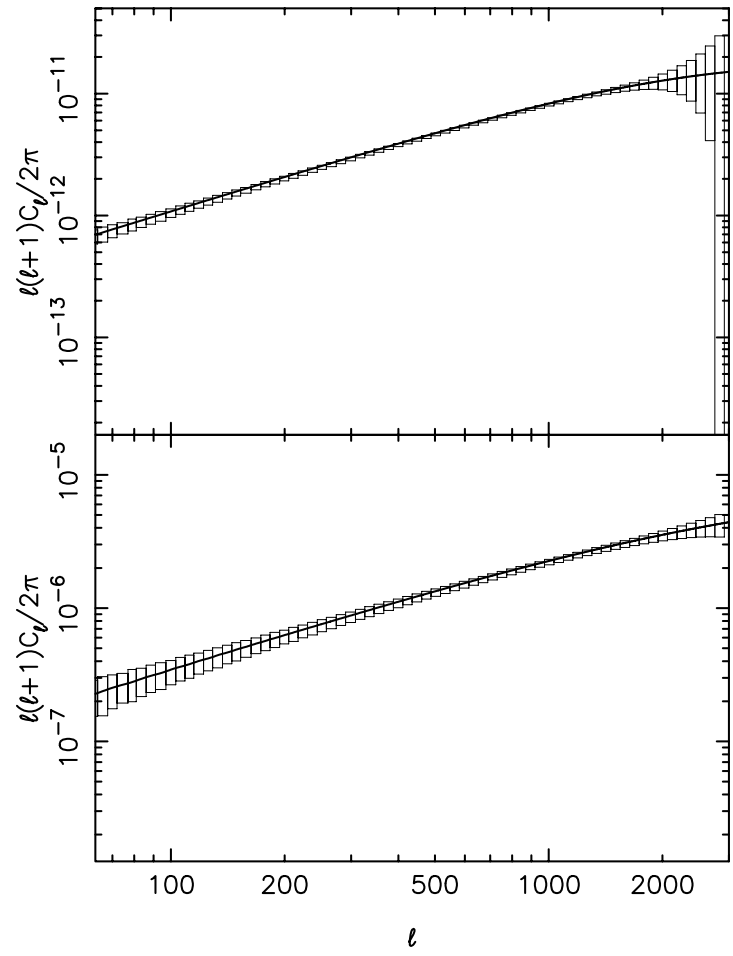

Fig. 6. Expected angular power spectrum of the SXRB-SZ correlation and corresponding correlation coefficient, with no separation of contributions in either redshift or mass-space. The error boxes are for the observation of the $25 \%$ of the sky with the DUET mission and include the Plank noise. Bin width is chosen to be $\Delta \log \ell=0.025$.

We now demonstrate the possibility that the cross power spectrum of SXRB and SZ map can be detected with upcoming CMB experiment such as Planck and X-ray measurement such as DUTE mission. To describe errors related to an SZ map, we follow the approach introduced by Cooray \& Hu (2000) and use the expected error on the SZ power spectrum based on multifrequency cleaning techniques. For the SXRB side, we make use of the X-ray catalog that is planned to be produced with the DUET mission. This survey is expected to cover $10000 \mathrm{deg}^{2}$ down to a flux limit of $5 \times 10^{-14} \mathrm{ergs} \mathrm{cm}^{-2} \mathrm{~s}^{-1}$. The errors on the cross power spectrum and the corresponding cross-correlation coefficient are calculated following the equations derived in Song et al. (2003).

We show in Fig. 6 the expected cross power spectrum and correlation coefficient to be detected by Planck and DUTE missions. It turns out that with these future experiments one can determine the correlation down to $\ell \approx 2000$ and to an accuracy of $\sim 0.04$. However, the current $\mathrm{CMB}$ data, such as those from the WMAP satellite, cannot be used to detect the crosscorrelation between SZ effect and SXRB because the fluctuations associated with the $\mathrm{SZ}$ effect is dominated by the primordial anisotropies of CMB. For reliable studies, a separate SZ map is a must and an opportunity for this is certainly available from the Planck mission. While we have assumed a smooth distribution of gas within halos, any nonstandard physical effect, such as cooling and preheating, will affect the cross-correlation. Though Planck and a similar X-ray catalog will present a first opportunity for a reliable detection of the 
cross-correlation between SZ and X-ray backgrounds, with targeted observations of small areas with better sensitivities, especially on the SZ side, we expect significant improvements and opportunities for understanding effects such as nongravitational heating.

\section{Discussion and conclusions}

Both X-ray emission and SZ effect arise from the WHIM gravitationally bound in massive clusters or large-scale structures. However, the two phenomena have very different response to the underlying gas distribution. The X-ray emission is more sensitive to the clumped gas structures (i.e. the central cores of clusters), while the SZ effect probes a much wider region of gas distribution out to virial radii of the systems. This behavior is reflected by the power spectra of their auto-correlation functions that are peaked at a larger $\ell$ for the SXRB and a smaller $\ell$ for the SZ map (see Fig. 1). Moreover, X-ray emission and SZ effect demonstrate different dependence on the distances of clusters from us. As a consequence, the major contribution to the SXRB comes from nearby clusters. This compares to the thermal SZ sky at small angular scales, which is dominated by high-redshift $(z>0.5)$ clusters. Therefore, the cross-correlation between the SXRB and SZ sky allows us to probe the distribution and evolution of the hot gas at intermediate angular scales and redshifts, as are shown by Fig. 2. Even though the cross-correlation coefficient is relatively mild $\sim 0.3$ (Fig. 5), Planck can allow a reliable detection of that out to a multipole of $\sim 3000$. Further improvements one can hope will be achieved in the post-Planck era, and detection of such a correlation would allow us to further understand the gas/baryon distribution and the certain physical properties. Actually, crosscorrelation between SXRB (or SZ) and galaxies have been extensively explored in literature. An corporation of these crosscorrelation and auto-correlation analyses of the SXRB may constitute a powerful tool to expose the evolution and distribution of the missing baryons in the universe. Of course, our theoretical predictions have been made without correcting for various spurious correlations. For example, most of the SXRB is actually generated by AGNs rather than diffuse sources like clusters and groups. Even if the contribution of AGNs can be nicely removed, the residuals may still be dominated by some nearby rich clusters (e.g. Diego et al. 2003). As for the SZ sky, potential sources of contaminations are the Milky Way, radio point sources and even radio halos of clusters (Cooray et al. 1998; Bouchet \& Gispert 1999; Holder 2002; Rubiño-Martín \& Sunyaev 2003; Zhou \& Wu 2003; etc.), provided that primary CMB anisotropies are successfully subtracted. Therefore, much work should be done to understand uncertainties in the detection of the SXRB-SZ cross correlation at small angular scales below $\sim 10$ arcmin in future experiments.

Acknowledgements. We gratefully acknowledge the valuable comments by an anonymous referee. This work was supported by the National Science Foundation of China, under Grant No. 10233040, and the Ministry of Science and Technology of China, under Grant No. NKBRSF G19990754.

\section{References}

Bouchet, F. R., \& Gispert, R. 1999, New Astron., 4, 443

Bryan, G. L., \& Norman, M. L. 1998, ApJ, 495, 80

Bryan, G. L., \& Voit, G. M. 2001, ApJ, 556, 590

Bullock, J. S., Kolatt, T. S., Sigad, Y., et al. 2001, MNRAS, 321, 559

Cen, R., \& Ostriker, J. P. 1999, ApJ, 514, 1

Cooray, A., Hu, W., \& Tegmark, M. 2000, ApJ, 540, 1

Cooray, A., \& Hu, W. 2000, ApJ, 534, 533

Cooray, A., \& Sheth, R. 2002, Phys. Rep., 372, 1

Cooray, A. R., Grego, L., Holzapfel, W. L., et al. 1998, AJ, 115, 1388

da Silva, A. C., Kay, S. T., Liddle, A. R., et al. 2001, ApJ, 561, L15

Davé, R., Cen, R., Ostriker, J. P., et al. 2001, ApJ, 552, 473

Diego, J., Silk, J., \& Sliwa, W. 2003, MNRAS, in press [astro-ph/0302268]

Fang, T., Marshall, H. L., Lee, J. C., Davis, D. S., \& Canizares, C. R. 2002, ApJ, 572, L127

Fukugita, M., Hogan, C. J., \& Peebles, P. J. E. 1998, ApJ, 503, 518

Ghigna, S., Moore, B., Governato, F., et al. 2000, ApJ, 544, 616

Jenkins, A., Frenk, C. S., White, S. D. M., et al. 2001, MNRAS, 321, 372

Holder, G. P. 2002, ApJ, 580, 36

Kaastra, J. S., Lieu, R., Tamura, T., Paerels, F. B. S., \& den Herder, J. W. 2003, A\&A, 397, 445

Komatsu, E., \& Seljak, U. 2002, MNRAS, 336, 1256

Mo, H.-J., \& White, S. D. M. 1996, MNRAS, 315, 356

Navarro, J. F., Frenk, C. S., \& White, S. D. M. 1995, MNRAS, 275, 720

Nevalainen, J., Lieu, R., Bonamente, M., \& Lumb, D. 2003, ApJ, 584, 716

Nicastro, F., Zezas, A., Drake, J., et al. 2002, ApJ, 573, 157

Nicastro, F., Zezas, A., Drake, J., et al. 2003, Nature, 421, 719

Ponman, T. J., Cannon, D. B., \& Navarro, J. F. 1999, Nature, 397, 135

Ponman, T. J., Sanderson, A. J. R., \& Finoguenov, A. 2003, MNRAS, 343,331

Raymond, J. C., \& Smith, B. W. 1977, ApJS, 35, 419

Rubiño-Martín, J. A., \& Sunyaev, R. A. 2003, MNRAS, 344, 1155

Scharf, C., Donahue, M., Voit, G. M., Rosati, P., \& Postman, M. 2000, ApJ, 528, L73

Soltan, A. M., Freyberg, M., \& Trumper, J. 2001, A\&A, 349, 354

Soltan, A. M., Freyberg, M., \& Hasinger, G. 2002, A\&A, 395, 475

Song, Y.-S., Cooray, A., Knox, L., \& Zaldarriaga, M. 2003, ApJ, 590, 664

Spergel, D. N., Verde, L., Peiris, H. V., et al. 2003, ApJS, 148, 175

Voit, G. M., \& Bryan, G. L. 2001, Nature, 414, 425

White, M., Hernquist, L., \& Springel, V. 2002, ApJ, 579, 16

Wu, X.-P., \& Xue, Y.-J. 2002, ApJ, 572, 19

Wu, X.-P., \& Xue, Y.-J. 2003, ApJ, 590, 8

Xue, Y.-J., \& Wu, X.-P. 2003, ApJ, 584, 34

Zappacosta, L., Mannucci, F., Maiolino, R., et al. 2002, A\&A, 394, 7

Zentner, A. R., \& Bullock, J. S. 2003, ApJ, in press [astro-ph/0304292]

Zhang, P.-J., \& Pen, U.-L. 2003 ApJ, 588, 704

Zhang, Y.-Y., \& Wu, X.-P. 2003, ApJ, 583, 529

Zhou, W., \& Wu, X.-P. 2003, ApJ, in press 